\title{
LC-Mass Spectrometry Analysis of N- and C-Terminal Boundary Sequences of Polypeptide Fragments by Limited Proteolysis
}

\author{
Justin G. Stroh, Pat Loulakis, Anthony J. Lanzetti, and Julie Xie* \\ PGRD-Groton Laboratories, Pfizer Inc., Groton, Connecticut, USA
}

Limited proteolysis is an important and widely used method for analyzing the tertiary structure and determining the domain boundaries of proteins. Here we describe a novel method for determining the $\mathrm{N}$ - and C-terminal boundary amino acid sequences of products derived from limited proteolysis using semi-specific and/or non-specific enzymes, with mass spectrometry as the only analytical tool. The core of this method is founded on the recognition that cleavage of proteins with non-specific proteases is not random, but patterned. Based on this recognition, we have the ability to determine the sequence of each proteolytic fragment by extracting a common association between data sets containing multiple potential sequences derived from two or more different mass spectral molecular weight measurements. Proteolytic product sequences derived from specific and non-specific enzymes can be accurately determined without resorting to the conventional time-consuming and laborious methods of SDS-PAGE and N-terminal sequencing analysis. Because of the sensitivity of mass spectrometry, multiple transient proteolysis intermediates can also be identified and analyzed by this method, which allows the ability to monitor the progression of proteolysis and thereby gain insight into protein structures. (J Am Soc Mass Spectrom 2005, 16, 38-45) (c) 2004 American Society for Mass Spectrometry

$\mathrm{P}$ roteins are dynamic systems containing structured domains linked by flexible loop/hinge regions. Flexibility is important for protein conformational changes that carry out specific functions, such as protein-ligand and protein-protein interactions [1-3]. Limited proteolysis is a widely used method to probe conformational changes, gain insights into protein tertiary structure, and to define domain boundaries and dynamics of proteins [4-6]. This approach, which utilizes proteases to cleave one or a limited number of peptide bonds in a protein, relies on the fact that mobile and flexible regions within a protein are more sensitive to protease digestion than organized protein structures, and thus, the structural and dynamic features of proteins can be defined $[7,8]$.

Various methods have been used to analyze the products of limited proteolysis. Routinely, after the target protein has been digested with sequence-specific or non-specific proteases, the resulting products are normally analyzed by gel electrophoresis to examine

Published online November 18, 2004

Address reprint requests to Dr. J. G. Stroh, PGRD-Groton Laboratories, Pfizer Inc., Groton, CT 06340, USA. E-mail: Justin_g_stroh@Groton. pfizer.com

* Readers can also contact Julie (Zhi) Xie, Protein \& Peptide Chemistry, EMS Discovery, Groton Laboratories, Pfizer, Inc., MS 8118W-112 Eastern Point Road, Groton, CT 06340. E-mail: zhi_xie@groton.pfizer.com. the fragment profile or to identify any band pattern changes of the protein derived from pretreatment of the protein under different conditions [9-11]. To obtain further information on the N- and C-terminal boundaries of proteolytic products, the bands can be analyzed by Western blotting [4] or by $\mathrm{N}$-terminal sequencing [5] to acquire amino acid sequences of the products. The C-terminus of the product is usually derived when the definitive $\mathrm{N}$-terminal sequence of the digested sequence is known and is combined with the molecular weight of the product estimated by gel analysis or measured by mass spectrometry. In a few cases, the C-terminus of the products have been determined directly by laborious and complicated C-terminal sequencing [12-14].

Mass spectrometry is a powerful tool for gaining molecular weight information on biological samples having molecular weights greater than $30 \mathrm{kDa}$ and is frequently used in conjunction with limited proteolysis to determine the molecular weight of the proteolyzed products [11, 15-24]. However, mass measurement alone does not produce a determination of the sequence of the proteolyzed products, since multiple subsequences usually will be retrieved fitting a given experimental mass, within experimental error. Thus, it is very difficult to determine the sequence of the product by mass spectrometry alone. 
(a)

\begin{tabular}{|c|c|c|c|c|c|c|}
\hline MHHHHHHSSG & LVPRGSYGHV & DIHEDPGPPP & PSPLVGLKPL & QLLEIKARGR & FGCVWKAQLM & 60 \\
\hline NDFVAVKIFP & LQDKQSWQSE & REIFSTPGMK & HENLLQFIAA & EKRGSNLEVE & LWLITAFHDK & 120 \\
\hline GSLTDYLKGN & IITWNELCHV & AETMSRGLSY & LHEDVPWCRG & EGHKPSIAHR & DFKSKNVLLK & 180 \\
\hline SDLTAVLADF & GLAVRFEPGK & PPGDTHGQVG & TRRYMAPEVL & EGAINFQRDA & FLRIDMYAMG & 10 \\
\hline$\underline{\text { LVLWELVSRC }}$ & KAADGPVDEY & MLPFEEEIGQ & HPSLEELQEV & VVHKKMRPTI & KDHWLKHPGL & \\
\hline AQLCVTIEEC & WDHDAEARLS & AGCVEERVSL & IRRSVNGTTS & DCLVSLVTSV & TNVDLPPKES & \\
\hline
\end{tabular}

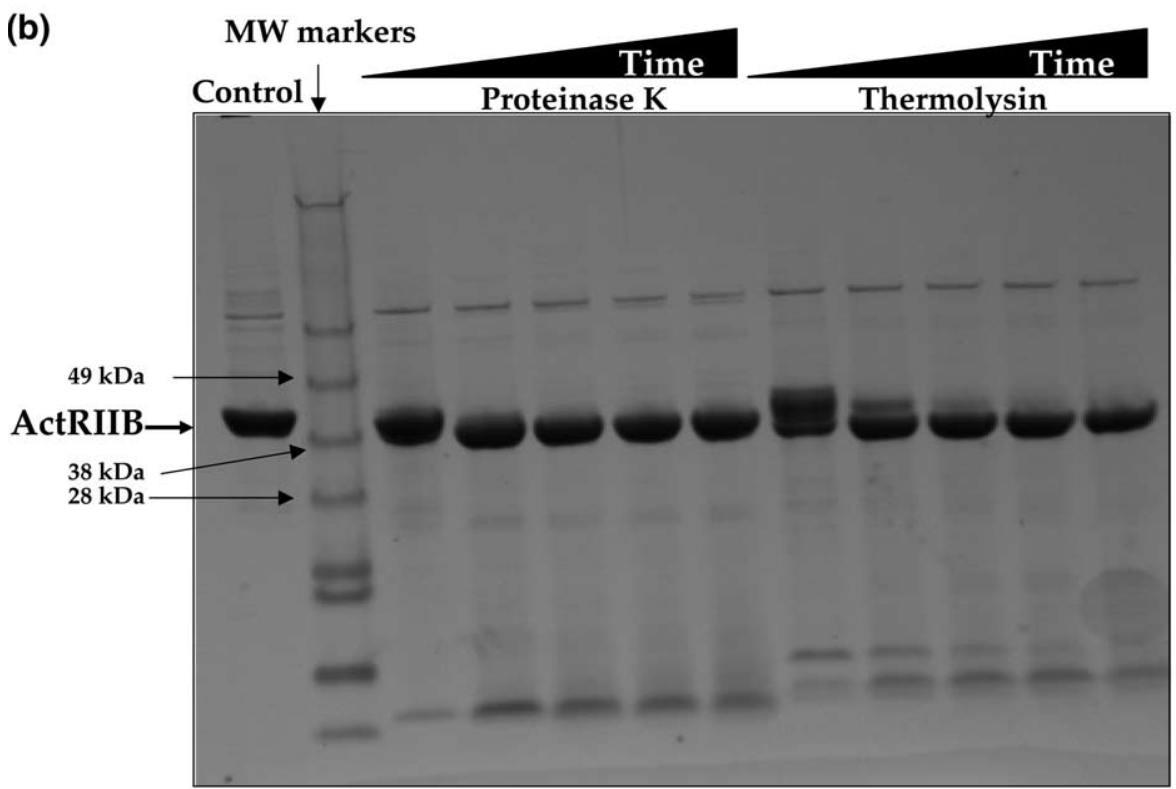

Figure 1. (a) Sequence of ActRIIBc. Underlined sequence (L34-T338) is the core structure as determined by limited proteolysis. (b) Gel of limited digestion of ActRIIBc. Lane 1 = undigested starting material. Lane $2=$ molecular weight markers. Lane $3=5$ min digestion with proteinase $\mathrm{K}$. Lane $4=30 \mathrm{~min}$ digestion with proteinase $\mathrm{K}$. Lane $5=60 \mathrm{~min}$ digestion with proteinase $\mathrm{K}$. Lane $6=$ $90 \mathrm{~min}$ digestion with proteinase $\mathrm{K}$. Lane $7=120 \mathrm{~min}$ digestion with proteinase $\mathrm{K}$. Lane $8=5 \mathrm{~min}$ digestion with thermolysin. Lane $9=30$ min digestion with thermolysin. Lane $10=60$ min digestion with thermolysin. Lane $11=90$ min digestion with thermolysin. Lane $12=120$ min digestion with thermolysin.

To counteract this limitation, specific endoproteases [15-21, 24-28] or exopeptidases [12-14], which only cut at specific amino acids or from the $\mathrm{N}$ - or C-terminus of the protein, are routinely used in combination with mass spectrometry to narrow the number of possibilities for a given observed molecular weight. However, the effort to define the structural domain can be compromised by the lack of the amino acid(s) required for the cleavage at the boundaries and the limited action of the exopeptidases at the $\mathrm{N}$ - or C-terminus. To determine the limited proteolysis products by non-specific proteases, additional sequence information derived either from multi-step traditional methods or subsequences defined with specific proteases treatment [22] is often required.

Therefore, the current analysis of limited proteolysis products is of restricted versatility. Even though mass spectrometry provides accurate and sensitive mass measurement, determination of the sequence of the proteolyzed products still routinely requires the tradi- tional time-consuming analysis steps and also demands significant amounts of sample. Here, we describe a new method using mass spectrometry as the only analytical tool for determination of $\mathrm{N}$ - and C-terminal boundary sequences of limited proteolysis products by non-specific enzymes.

\section{Experimental}

\section{Limited Proteolysis}

A His-tagged ActRIIB construct (MW 40,772 Da) containing the catalytic domain [29] of the kinase was purified by NiNTA affinity column. The purified protein with a concentration of $1 \mathrm{mg} / \mathrm{ml}$ was incubated with a series of specific or non-specific proteases in a digestion buffer containing $20 \mathrm{mM}$ Tris- $\mathrm{HCl}(\mathrm{pH} 8.0)$, $150 \mathrm{mM} \mathrm{NaCl}, 5 \mathrm{mM}$ DTT, and $10 \%$ glycerol at $37^{\circ} \mathrm{C}$. The enzyme to substrate ratio (wt/wt) was 1:500 for trypsin (Roche, Indianapolis, IN), 1:2000 for proteinase 
Table 1. Theoretical sub-sequences of ActRIIBc that fit the observed molecular weight from a 30-min trypsin digest

\begin{tabular}{|c|c|c|lll|}
\multicolumn{1}{c}{ Mass } & DeV & From-To & \multicolumn{2}{c|}{ Sequence } \\
\hline 39083 & 0.1 & $6-353$ & HH & HHSSGLVPRGSYGHVDIHED....SVNGTTSDCLVSLVTSVTNV & DL \\
39081 & -2.0 & $3-349$ & MH & HHHHHSSGLVPRGSYGHVDI....LIRRSVNGTTSDCLVSLVTS & VT \\
39079 & -3.9 & $15-362$ & PR & < GSYGHVDIHEDPGPPPPSPL....LVSLVTSVTNVDLPPKESSI> \\
\hline
\end{tabular}

Column 1 is the theoretical mass, Column 2 is the difference between the observed and calculated mass, Column 3 is the subsequence position, and Column 4 is the actual sub-sequence. In Column 4, the first two and last two (red highlighted amino acids) are the two N-terminal and C-terminal amino acids, respectively, that are beyond the sub-sequence.

K (Roche), and 1:50 for thermolysin (Sigma, St. Louis, $\mathrm{MO}$ ). To the thermolysin digest, $5 \mathrm{mM} \mathrm{CaCl}_{2}$ was supplemented for the protease to function. $50 \mu \mathrm{L}$ aliquots were removed from the $500 \mu \mathrm{L}$ digestion samples at 5, 30, 60, 90, and $120 \mathrm{~min}$. Digestion was terminated by the addition of PMSF for trypsin and proteinase $\mathrm{K}$, and with $10 \mathrm{mM}$ EDTA for thermolysin.

\section{Liquid Chromatography/Mass Spectrometry Analysis}

From each of the $50 \mu \mathrm{L}$ aliquot of digested material, a $5 \mu \mathrm{L}$ sample was extracted that was diluted with $25 \mu \mathrm{L}$ of $0.1 \%$ TFA in water to make a total of $30 \mu \mathrm{L}$ of solution. Each $30 \mu \mathrm{L}$ sample was placed in a 96 well plate that was inserted into a Famos (LC Packings, San Francisco, CA) autosampler. Samples were chilled to $5{ }^{\circ} \mathrm{C}$ until injection. Then, a $10 \mu \mathrm{L}$ aliquot of each sample was injected on-column to a Vydac C-18 capillary column $(0.3 \times 50 \mathrm{~mm})$. An Applied Biosystems (Foster City, CA) 140D solvent delivery module was used to deliver an HPLC gradient of $10-80 \% \mathrm{~B}$ over $30 \mathrm{~min}$ (A = $0.02 \%$ TFA in $95 \%$ water $/ 5 \%$ acetonitrile, B = acetonitrile) at a flow rate of $10 \mu \mathrm{L} / \mathrm{min}$. Samples eluting the column flowed through an Applied Biosystems single wavelength UV detector operating at $220 \mathrm{~nm}$. The UV detector was fitted with an LC Packings (San Francisco, CA) U-Z View flow cell. Samples exiting the UV detector entered a Micromass (Altrincham, UK) Ultima triple quadrupole mass spectrometer employing electrospray ionization and operating in the single quadrupole mode. The instrument was operated in the positive ion mode using a capillary voltage of $3.31 \mathrm{kV}$, a cone voltage of $110 \mathrm{~V}$, a source temperature of $80^{\circ} \mathrm{C}$, a desolvation temperature of $120^{\circ} \mathrm{C}$, a cone gas flow of nitrogen equal to $123 \mathrm{~L} / \mathrm{h}$, and a desolvation flow of $710 \mathrm{~L} / \mathrm{h}$. The instrument was scanned over the $\mathrm{m} / \mathrm{z}$ range of $350-2400$ at a rate of $5.2 \mathrm{~s}$ per scan. Data was collected over a $30-\mathrm{min}$ period. Raw data was deconvoluted to produce molecular weight information of the protein and peptide fragments using MaxEnt, which is the software provided by Micromass for deconvoluting multiply charged data. The data was processed to approximately $30 \%$ convergence. Other parameters for MaxEnt were as suggested by the manufacturer.

\section{Data Analysis}

A table of molecular weights was prepared for each digestion time point and compared against other time points for the same digestion enzyme. For the molecular weight of each product formed in digestion, a list of possible sub-sequences of the original protein was calculated using the program GPMAW, version 4.2 (Lighthouse Data, Odense, Denmark). Subsequences were calculated with a theoretical error of $\pm 0.03 \%$. Each set of subsequences was compared with adjacent molecular weight subsequences in order to determine if there was an association between the two molecular weights. Comparison of the data points occurred and the common data point was determined.

\section{N-Terminal Sequencing}

Automated Edman sequencing was performed on an Applied Biosystems Procise Sequencer, Model 494. Data were collected and analyzed using the Applied Biosystems Model 610A software.

\section{Results and Discussion}

We used the catalytic domain of the activin receptor type II B (ActRIIBc), a Thr/Ser kinase, as an example to demonstrate this method. The construct lacked the first

Table 2. Theoretical sub-sequences of ActRIIBc that fit the observed molecular weight from a 30-min thermolysin digest

\begin{tabular}{|c|c|c|c|c|}
\hline Mass & Dev & From-To & & Sequence \\
\hline 35066 & 0.5 & $16-325$ & $R G$ & SYGHVDIHEDPGPPPPSPLV....TIEECWDHDAEARLSAGCVE \\
\hline 35066 & 0.6 & $40-348$ & KP & LQLLEIKARGRFGCVWKAQL....SLIRRSVNGTTSDCLVSLVT SV \\
\hline 35062 & -3.4 & $33-347$ & LK & PLQLLEIKARGRFGCVWKAQ....VSLIRRSVNGTTSDCLVSLV TS \\
\hline 35069 & 3.7 & $28-336$ & PG & PPPPSPLVGLKPLQLLEIKA....ARLSAGCVEERVSLIRRSVN \\
\hline 35061 & -4.4 & $35-343$ & PL & VGLKPLQLLEIKARGRFGCV....VEERVSLIRRSVNGTTSDCL VS \\
\hline 35061 & -4.4 & $36-344$ & LV & GLKPLQLLEIKARGRFGCVW....EERVSLIRRSVNGTTSDCLV SL \\
\hline 35061 & -4.4 & $34-342$ & SP & LVGLKPLQLLEIKARGRFGC....CVEERVSLIRRSVNGTTSDC LV \\
\hline 35070 & 4.5 & $7-316$ & $\mathrm{HH}$ & HSSGLVPRGSYGHVDIHEDP....HPGLAQLCVTIEECWDHDAE AR \\
\hline
\end{tabular}

Explanation of the column of this table is the same as given in Table 1. 

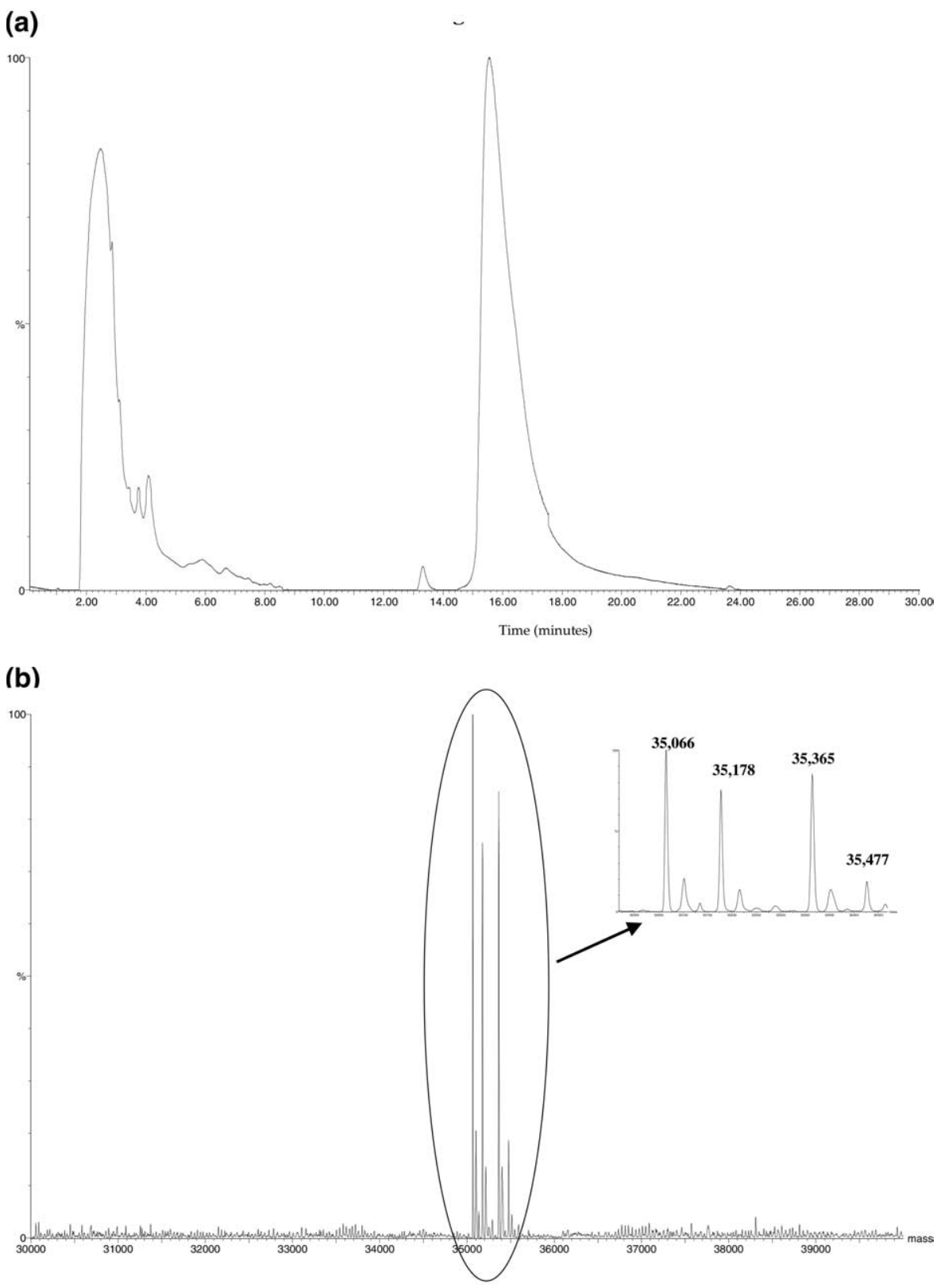

Figure 2. (a) HPLC/UV of 30-min thermolysin digest. (b) Deconvoluted electrospray mass spectrum of 30-min thermolysin digest. Taken from HPLC/UV peak in Figure 2a at a retention time of 16 min.

166 amino acids of the enzyme and was His-tagged at the N-terminus. The molecular weight of the ActRIIBc (167-512) was 40,775 Da (average) when measured by LC-MS and matched the theoretical mass $(40,772 \mathrm{Da})$ of the construct with the addition of acetylation at the $\mathrm{N}$-terminal amino acid. The position of the amino acids of ActRIIBc in the following text was defined as showed in Figure 1a. Limited proteolysis of the protein was performed with trypsin and two non-specific enzymes, thermolysin and proteinase $\mathrm{K}$. Aliquots of the reaction mixture were extracted at different times (5 to $120 \mathrm{~min}$ ) and the products were analyzed by SDS-PAGE (Figure $1 b$ ) and electrospray ionization LC/MS as described in the Experimental Methods. Cleavage with trypsin gave one peak in the high mass range at 39,082.8 $\pm 7.8 \mathrm{Da}$ (data not shown). Three possible subsequences (Table 1) were calculated that matched the observed mass within experimental error. Since trypsin cuts only at the Cterminus of Lys/Arg, the only subsequence to match this requirement was G15-I362, which resulted from cleavage at R14 and contains the C-terminus of the protein. Thus, selective action of specific proteases helps to determine the correct subsequence from multiple possibilities and this is one of the major reasons that trypsin has been widely used in conjunction with mass spectrometry to analyze sequences derived from limited proteolysis.

On the other hand, non-selective enzymes such as 


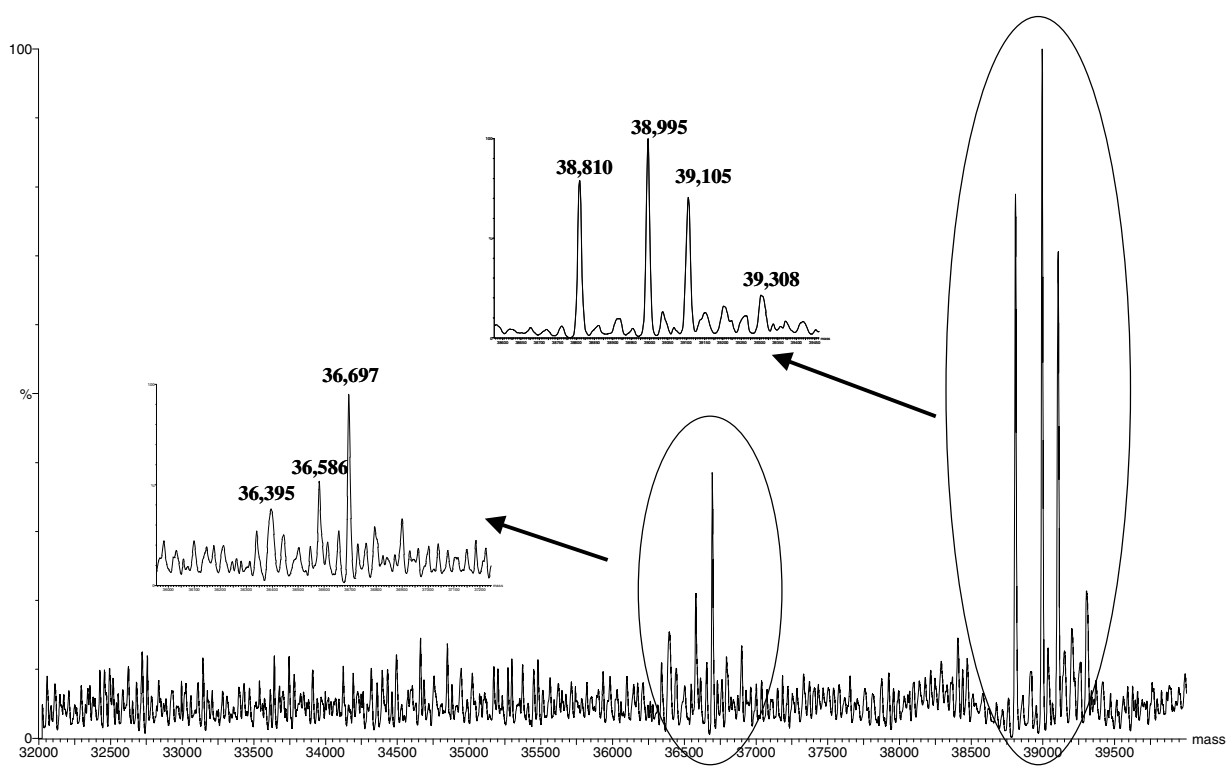

Figure 3. Deconvoluted electrospray mass spectrum of 5-min proteinase K digest of ActRIIBc.

thermolysin and proteinase $\mathrm{K}$ are less successful when used only in conjunction with mass spectrometry, since they can digest a much broader range of amino acids. For example, after the ActRIIBc was digested with thermolysin for $30 \mathrm{~min}$, a mass of 35,066 Da was observed using LC-MS analysis of the proteolysis products. Even when the mass spectral experimental error was reduced to $0.015 \%$ (or $\pm 5.3 \mathrm{Da}$ ), a total of eight possible subsequences were retrieved based on the observed mass within the experimental error (Table 2). Furthermore, three of the eight possibilities have the same exact mass $(35,061 \mathrm{Da})$, as they are isomers of each other (V35-L343, G36-V344, and L34-C342). Without additional sequence information, it is not possible to determine which of the eight possibilities is the correct one. Therefore, to determine the sequence of proteolytic products of non-specific proteases, additional steps, such as $\mathrm{N}$ - terminal or C-terminal sequencing, or comparison with other known subsequences, are normally required. However, we demonstrate here a method that uses mass spectrometry as the only measurement device, and from mass spectral measurements the particular subsequence can be unambiguously identified derived from proteolytic cleavage by non-specific enzymes.

The fundamental premise of this method is the recognition that the cleavage of proteins by non-specific enzymes (such as thermolysin and proteinase $\mathrm{K}$ ) is not random, but patterned. As an example, the HPLC/UV of a fast gradient LC/MS derived from a 30-min digest of ActRIIBc with thermolysin is shown in Figure 2a. The protein peak with a retention time of 16 min gave the deconvoluted (molecular weight) electrospray mass spectrum shown in Figure 2b. Deconvoluted molecular weights were processed over the mass range $30,000-$ $41,000 \mathrm{Da}$. It appeared that this is not a spectrum of random molecular weights; on the contrary, all of the molecular weight peaks are congregated in a narrow mass range, suggesting that these mass species are related. This is again demonstrated in the deconvoluted mass spectrum of the 5-min proteinase $\mathrm{K}$ digestion of ActRIIBc (Figure 3), which shows two different congregations of peaks.

The question is how these molecular weight peaks relate to each other. As described above, it is not possible, based on a single mass measurement to determine which of the eight possibilities is the correct sequence for the observed mass of 35,066 Da (Table 2). However, when the possible subsequences matching all of the measured masses in a congregation of peaks are analyzed simultaneously, a pattern emerges showing the cleavage footprint of the protease at a sensitive site. As an example, the masses shown in Figure $2 b$ (35,066 $\mathrm{Da}, 35,178 \mathrm{Da}, 35,365 \mathrm{Da}$, and 35,477 Da) produce four sets of data of potential subsequences as shown in Figure 4. A common association running through the data sets was identified as the sequential cleavage using non-specific proteases (Figure 4). Polypeptides L34C342, L34-343L, L34-345S, and L34-L346L were recognized from each of the data sets that showed the footprint of thermolysin cleavage. Through this analysis, the consensus sequence was identified from multiple data sets (underlined in Figure 4). A table of all cleavage products at various digestion time points can also be determined to demonstrate the progression of digestion by thermolysin (Table 3). A similar strategy had been successfully applied to determine the $\mathrm{N}$ - and C-terminal boundary sequences of products digested by proteinase $\mathrm{K}$ at various time-points as shown Figure $5 \mathrm{a}$ and $\mathrm{b}$ and Table 4.

In addition, the 90-min digests of ActRIIBc with thermolysin and proteinase $\mathrm{K}$ were also analyzed by 


\begin{tabular}{ll}
\multicolumn{3}{l}{ Observed mass: 35066} \\
Mass (Da) & From- \\
35066 & $16-$ \\
35066 & $40-$ \\
35062 & $39-$ \\
$\mathbf{3 5 0 6 1}$ & $\mathbf{3 4 -}$ \\
\hline 35061 & $35-$ \\
35061 & $36-$ \\
35069 & $28-$ \\
35070 & $7-$ \\
& \\
Observed mass: 35178 \\
Mass (Da) & From- \\
35176 & $1-$ \\
35181 & $49-$ \\
$\mathbf{3 5 1 7 5}$ & $\mathbf{3 4 -}$ \\
35182 & $46-$
\end{tabular}

\begin{tabular}{ll}
$\begin{array}{c}5.3 \mathrm{Da} \\
\text { To }\end{array}$ & \\
325 & R \\
348 & K \\
347 & L \\
$\mathbf{3 4 2}$ & S \\
\hline 343 & P \\
344 & L \\
336 & P \\
316 & H
\end{tabular}

Sequence

SYGHVDIHED.... EARLSAGCVE ER KP LQLLEIKARG.... TSDCLVSLVT SY LK PLQLLEIKAR.... TTSDCLVSLV TS SP LVGLKPLQLL... RSVNGTTSDC L. PL VGLKPLQLLE.... VNGTTSDCL VS LV GLKPLQLLEI.... VNGTTSDCLV SL PG PPPPSPLVGL.... RVSLIRRSVN GT HH HSSGLVPRGS.... IEECWDHDAE AR

Observed mass: $35365 \quad 5.3$ Da

$\begin{array}{lll}\text { Mass (Da) } & \text { From- } & \text { T } \\ 35364 & 17- & 3 \\ 35362 & 1- & 3 \\ 35361 & 35- & 3 \\ 35361 & 36- & 3 \\ \mathbf{3 5 3 6 1} & \mathbf{3 4 -} & \mathbf{3} \\ 35361 & 11- & 3 \\ 35361 & 8- & 3\end{array}$

$5.3 \mathrm{Da}$ To
310
359

(

Sequence

MHHHHHHSSG.... AQLCVTIEEC WD AR GRFGCVWKAQ.... VTNVDLPPKE SS SP LVGLKPLOLل. SVNGTTSDCL V

EI KARGRFGCVW.... VTSVTNVDLP PK

3 Da

\begin{tabular}{l}
$5.3 \mathrm{Da}$ \\
To \\
328 \\
311 \\
346 \\
347 \\
$\mathbf{3 4 5}$ \\
\hline 323 \\
320
\end{tabular}

Sequence

GS YGHVDIHEDP.... LSAGCVEERV SL MHHHHHHSSG.... QLCVTIEECW DH MHHHHHHSSG.... QLCVTIEECW DI
VGLKPLQLLE... GTTSDCLVSL VT PL VGLKPLQLLE.... GTTSDCLVSL VT
LV GLKPLQLLEI... TTSDCLVSLV TS SP IVGLKPLQLL... NGTTSDCLVS LV HH SSGLVPRGSY.... WDHDAEARLS AG

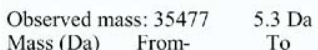

35477 1-

$35476 \quad 20-$

35476

35478

$\begin{array}{lll}35474 & 34- & 346 \\ 35473 & 27- & 340\end{array}$

$35482 \quad 4-\quad 316$

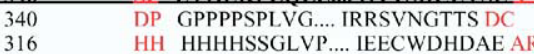

Figure 4. Calculation of all theoretical sub-sequences from ActRIIBc for each individual mass shown in Figure 2b, within experimental error.

conventional SDS-PAGE followed by transferring to a PVDF membrane and $\mathrm{N}$-terminal sequencing. The amino acid sequence LVGLK was identified at the $\mathrm{N}$-terminal of the proteolytic fragment that resulted from the thermolysin digestion, while amino acid sequences IHEDP and EDPGP were identified as the $\mathrm{N}$-terminal amino acids for the proteinase $\mathrm{K}$ digestion products (data not shown). These findings were consistent with the results obtained using the method described in this paper that proteolytic fragments containing L34 as the N-terminus were present in the 90-min thermolysin digests (Table 3 ) and fragments containing I22 and E24 as the N-terminal amino acids were present in the 90-min proteinase $\mathrm{K}$ digests (Table 4). Thus, without traditional gel electrophoresis and $\mathrm{N}$-terminal sequencing, we are able to determine the sequence of (a)

\begin{tabular}{|c|c|c|c|}
\hline Observed $\mathrm{m}$ & ss: 36395 & $5.5 \mathrm{Da}$ & \\
\hline Mass (Da) & From- & To & Sequence \\
\hline 36394 & $20-$ & 341 & GH VDIHEDPGPP.... RRSVNGTTSD CL \\
\hline$\underline{36396}$ & $22-$ & 343 & _VD_IHEDPGPPPP.... SVNGTTSDCL VS \\
\hline 36398 & $21-$ & 342 & HV DIHEDPGPPP.... RSVNGTTSDC LV \\
\hline 36391 & 27- & 349 & DP GPPPPSPLVG.... SDCLVSLVTS VT \\
\hline Observed m & ss: 36586 & $\pm 5.5 \mathrm{Da}$ & \\
\hline Mass (Da) & From- & To & Sequence \\
\hline 36587 & 9- & 332 & HS SGLVPRGSYG.... CVEERVSLIR RS \\
\hline 36587 & $32-$ & 355 & PP SPLVGLKPLQ.... LVTSVTNVDL PP \\
\hline 36587 & 26- & 350 & ED PGPPPPSPLV.... DCLVSLVTSV TN \\
\hline 36585 & $37-$ & 359 & VG LKPLQLLEIK.... VTNVDLPPKE SS \\
\hline 36588 & $1-$ & 323 & MHHHHHHSSG.... DAEARLSAGC VE \\
\hline 36588 & $18-$ & 341 & SY GHVDIHEDPG.... RRSVNGTTSD CL \\
\hline 36590 & 14 & 336 & VP RGSYGHVDIH.... RVSLIRRSVN GT \\
\hline$\underline{36582}$ & 22. & 345 & VD IHEDPGPPPP.... NGTTSDCLVS LV \\
\hline 36582 & $23-$ & 346 & DI HEDPGPPPPS.... GTTSDCLVSL VT \\
\hline 36591 & 27- & 351 & DP GPPPPSPLVG.... CLVSLVTSVT NV \\
\hline Obser & ss: 36697 & $\pm 5.5 \mathrm{Da}$ & \\
\hline Mass (Da) & From- & To & Sequence \\
\hline 36697 & $21-$ & 345 & HV DIHEDPGPPP.... NGTTSDCLVS LV \\
\hline 36695 & 22. & 346 & _VD IHEDPGPPPP.... GTTSDCLVSL VT \\
\hline 36694 & 33- & 357 & PS PLVGLKPLQL.... TSVTNVDLPP KE \\
\hline 36693 & $15-$ & 339 & PR GSYGHVDIHE.... LIRRSVNGTT SD \\
\hline 36702 & $25-$ & 350 & HE DPGPPPPSPL.... DCLVSLVTSV TN \\
\hline
\end{tabular}

(b)

\begin{tabular}{|c|c|c|c|c|}
\hline \multicolumn{2}{|c|}{ Observed mass: 38810} & $\pm 6.2 \mathrm{Da}$ & \multirow{2}{*}{\multicolumn{2}{|c|}{ Sequence }} \\
\hline Mass (Da) & From- & To & & \\
\hline 38809 & 8- & 353 & $\mathrm{HH}$ & SSGLVPRGSY.... VSLVTSVTNV DL \\
\hline 38807 & 5- & 349 & $\mathrm{HH}$ & HHHSSGLVPR.... SDCLVSLVTS VT \\
\hline 38806 & $11-$ & 355 & & LVPRGSYGHV.... LVTSVTNVDL PP \\
\hline$\underline{38805}$ & 1. & 343 & & MHHHHHHSSG.... SVNGTTSDCL VS \\
\hline \multicolumn{2}{|c|}{ Observed mass: 38995} & 6.2 Da & & \\
\hline Mass (Da) & From- & To & & Sequence \\
\hline 38994 & $3-$ & 348 & MH & HHHHHSSGLV.... TSDCLVSLVT SV \\
\hline 38991 & 1. & 345 & & MHНHНHHSSG.... NGTTSDCLVS LV \\
\hline 39000 & $11-$ & 357 & SG & LVPRGSYGHV.... TSVTNVDLPP KE \\
\hline \multicolumn{2}{|c|}{ Observed mass: 39105} & $\pm 6.3 \mathrm{Da}$ & & \\
\hline Mass (Da) & From- & To & & Sequence \\
\hline 39104 & 1. & 346 & & MHHHHHHSSG.... GTTSDCLVSL VT \\
\hline \multicolumn{2}{|c|}{ Observed mass: 39308} & $\pm 6.3 \mathrm{Da}$ & & \\
\hline Mass (Da) & From- & To & & Sequence \\
\hline 39311 & 6- & 355 & $\mathrm{HH}$ & HHSSGLVPRG.... LVTSVTNVDL PP \\
\hline 39304 & 1. & 348 & & MHHHHHHSSG.... TSDCLVSLVT SV \\
\hline 39314 & $10-$ & 359 & SS & GLVPRGSYGH.... VTNVDLPPKE SS \\
\hline
\end{tabular}

Figure 5. (a) and (b) Calculation of all theoretical sub-sequences from ActRIIBc for each individual mass shown in Figure 3, within experimental error.

products of limited proteolysis by non-specific enzymes. Since non-specific proteases do not require specific amino acids for digestion, they are likely to be

Table 3. Thermolysin digest data

\begin{tabular}{|c|c|c|c|c|c|}
\hline $5 \mathrm{~min}$ & $30 \mathrm{~min}$ & $60 \mathrm{~min}$ & $90 \mathrm{~min}$ & $120 \mathrm{~min}$ & Range \\
\hline $35,063(4)$ & $35,066(1)$ & $35,066(1)$ & $35,067(1)$ & $35,068(1)$ & $34-342$ \\
\hline $35,176(2)$ & $35,178(3)$ & $35,179(2)$ & $35,178(2)$ & $35,178(2)$ & $34-343$ \\
\hline $35,363(1)$ & $35,365(2)$ & $35,365(3)$ & $35,365(3)$ & $35,368(3)$ & $34-345$ \\
\hline 35,474 & $35,477(4)$ & $35,477(4)$ & & & $34-346$ \\
\hline 37,577 & & & & & $11-343$ \\
\hline 37,764 & & & & & $11-345$ \\
\hline 38,812 & & & & & $2-345$ \\
\hline $38,994(3)$ & & & & & $1-345$ \\
\hline 39,107 & & & & & $1-346$ \\
\hline
\end{tabular}

Column 1 = observed digest molecular weights after 5 -minutes of digestion. Column $2=$ as in 1 after 30 -minutes of digestion. Column $3=60$-min, $4=90 \mathrm{~min}$, and $5=120 \mathrm{~min}$. Column 6 is the sequences range identified for each observed molecular weight in the first five columns. Numbers in parentheses correspond to relative peak height. (1) = highest peak, $(2)=$ second highest peak, etc. 
Table 4. Proteinase $\mathrm{K}$ digest data

\begin{tabular}{|c|c|c|c|c|c|}
\hline $5 \mathrm{~min}$ & $30 \mathrm{~min}$ & $60 \mathrm{~min}$ & $90 \mathrm{~min}$ & $120 \mathrm{~min}$ & Range \\
\hline & & $35,634(4)$ & $35,632(4)$ & $35,632(3)$ & $24-338$ \\
\hline & $35,879(4)$ & $35,881(3)$ & $35,882(1)$ & $35,881(1)$ & $22-338$ \\
\hline & $36,069(3)$ & $36,070(2)$ & $36,070(2)$ & $36,071(2)$ & $22-340$ \\
\hline 36,395 & $36,400(1)$ & $36,401(1)$ & $36,401(3)$ & $36,401(4)$ & $22-343$ \\
\hline 36,586 & $36,586(2)$ & & & & $22-345$ \\
\hline $36,697(4)$ & & & & & $22-346$ \\
\hline $38,810(2)$ & & & & & $1-343$ \\
\hline $38,995(1)$ & & & & & $1-345$ \\
\hline 39,105 (3) & & & & & $1-346$ \\
\hline 39,308 & & & & & $1-348$ \\
\hline
\end{tabular}

Explanation of columns identical to that of Table 3.

more continuous and efficient in cleaving flexible regions of a protein compared with specific proteases that may be hindered by the absence of the specific amino acid in an accessible region of a protein. Thus, this rapid LC-MS analysis of limited proteolysis products by nonspecific proteases provides a more effective tool to probe and determine the domain boundaries of proteins.

The accuracy of the mass determination is critical, since the number of potential polypeptide sequences that match a given measured mass grows proportionally with experimental error. A large experimental error makes the identification of the common association between data sets difficult, because it compromises our ability to determine the single correct sequence from a list of many polypeptides matching the measured mass.

Because this method employs LC-MS, it is a sensitive tool for the analysis of minor transient proteolytic intermediates and is useful for monitoring the progression of proteolysis. Table 3 shows the proteolytic products that are identified during the time course of digestion of ActRIIBc with thermolysin. Multiple Nand C-terminally truncated fragments are identified after 5-min of incubation of ActRIIBc with thermolysin. Some products are transient and are further cleaved (e.g., H2-S345 and L11-S345), while others are relatively stable even when treated for up to 2 h (e.g., L34-C342 and L34-L343). Combining the digestion data of thermolysin and proteinase K (Figures 4 and 5), we determine that the polypeptide containing L34-T338 of ActRIIBc is relatively resistant to proteases digestion and composes a compact core structure of the kinase. The ability to analyze and identify transient intermediates and relatively stable polypetides during the course of digestion allows us to track the footprints of nonspecific proteases, define domain boundaries, and gain more insight to the dynamic features of the protein.

Another important feature of this method is robustness. With traditional methods, the probability of obtaining sequence information of the proteolytic products is often compromised by the resolution and sensitivity of SDS-PAGE and N-terminal sequencing. It often requires an optimization process to either resolve digestion products or identify a set of digestion conditions that result in only a very few cleavage products accumulating for successful $\mathrm{N}$-terminal sequencing. As shown in Figure 4, at least four mass species were detected using LC-MS, though the digestion products were barely separated by traditional gel electrophoresis, which made downstream $\mathrm{N}$-terminal sequencing analysis challenging. However, with the sensitivity and accuracy of the current method, sequences of the proteolytic products can be successfully determined during the course of digestion (Figures 4, 5 and Tables 3, 4) even if the products are transient and within a narrow mass range. Although an LC/MS run time of $30 \mathrm{~min} /$ sample was used in the current set of experiments, we have found that ballistic gradients employing a 10-min LC/MS run time (data not shown) are quite sufficient for this analysis. With optimization, the speed can be further improved.

Thus, this method provides a rapid, simple, accurate, and robust analysis for determining the sequences of limited proteolysis products using non-specific proteases. Because of the sensitivity of LC-MS, only a very small quantity of protein is required for analysis. The process could be automated in a high throughput format and be applied to define domain boundaries of proteins for construct design for structure studies and to probe detailed conformational changes of proteins when exposed to various treatments, such as activation or suppression.

\section{Conclusions}

We developed a rapid and accurate LC-MS method for the analysis and determination of product sequences of limited proteolysis using non-specific enzymes. The sequences of the proteolysis products at a given time point can be determined based solely on mass measurement and the progressive action of a non-specific protease. This finding eliminates the dependence on the traditional methods of gel electrophoresis and N-terminal sequencing, which often compromise the accuracy and sensitivity empowered by mass spectrometry. 


\section{References}

1. Todd, A. E.; Orengo, C. A.; Thornton, J. M. Evolution of Protein Function, from a Structural Perspective. Curr. Opin. Chem. Biol. 1999, 3(5), 548-556.

2. Kumar, S.; Ma, B.; Tsai, C. J.; Sinha, N.; Nussinov, R. Folding and Binding Cascades: Dynamic Landscapes and Population Shifts. Prot. Sci. 2000, 9(1), 10-19.

3. Bennett, W. S.; Huber, R. Structural and Functional Aspects of Domain Motions in Proteins. CRC Crit. Rev. Biochem. 1984, 15(4), 291-384.

4. Oren, D. A.; Li, Y.; Volovik, Y.; Morris, T. S.; Dharia, C.; Das, K.; Galperina, O.; Gentz, R.; Arnold, E. Structural Basis of BLYS Receptor Recognition. Nat. Struct. Biol. 2002, 9(4), $288-$ 292.

5. Monti, M.; Principe, S.; Giorgetti, S.; Mangione, P.; Merlini, G.; Clark, A.; Bellotti, V.; Amoresano, A.; Pucci, P. Topological Investigation of Amyloid Fibrils Obtained from $\beta 2$-Microglobulin. Prot. Sci. 2002, 11(10), 2362-2369.

6. Yang, S. A.; Klee, C. Study of Calcineurin Structure by Limited Proteolysis. Methods Mol. Biol. 2002, 172, 317-334.

7. Polverino de Laureto, P.; De Filippis, V.; Di Bello, M.; Zambonin, M.; Fontana, A. Probing the Molten Globule State of $\alpha$-Lactalbumin by Limited Proteolysis. Biochemistry 1995, 34(39), 12596-12604.

8. Fontana, A.; Fassina, G.; Vita, C.; Dalzoppo, D.; Zamai, M.; Zambonin, M. Correlation Between Sites of Limited Proteolysis and Segmental Mobility in Thermolysin. Biochemistry 1986, 25(8), 1847-1851.

9. Zhang, B.; Peng, Z. Y. Structural Consequences of TumorDerived Mutations in P16INK4a Probed by Limited Proteolysis. Biochemistry 2002, 41(20), 6293-6302.

10. Henn, A.; Shi, S. P.; Zarivach, R.; Ben-Zeev, E.; Sagi, I. The RNA Helicase DbpA Exhibits a Markedly Different Conformation in the ADP-Bound State When Compared with the ATP- or RNA-Bound States. J. Biol. Chem. 2002, 277(48), $46559-4665$.

11. Chen, G. Q.; Sun, Y.; Jin, R.; Gouaux, E. Probing the Ligand Binding Domain of the GluR2 Receptor by Proteolysis and Deletion Mutagenesis Defines Domain Boundaries and Yields a Crystalizable Construct. Prot. Sci. 1998, 7(12), 2623-2630.

12. Borowski, P.; Kuehl, R.; Mueller, O.; Hwang, L. H.; Schulze zur Wiesch, J.; Schmitz, H. Biochemical Properties of a Minimal Functional Domain with ATP-Binding Activity of the NTPase/Helicase of Hepatitis C Virus. Eur. J. Biochem. 1999, 266(3), 715-723.

13. Stoffer, B.; Frandsen, T. P.; Busk, P. K.; Schneider, P.; Svendsen, I.; Svensson, B. Production, Purification, and Characterization of the Catalytic Domain of Glucoamylase from Aspergillus niger. Part 1. Biochem. J. 1993, 292, 197-202.

14. Villanueva, J.; Villegas, V.; Querol, E.; Aviles, F. X.; Serrano, L. Protein Secondary Structure and Stability Determined by Combining Exoproteolysis and Matrix-Assisted Laser Desorption/Ionization Time-of-Flight Mass Spectrometry. J. Mass Spectrom. 2002, 37(9), 974-984.

15. Leite, J. F.; Amoscato, A. A.; Cascio, M. Coupled Proteolytic and Mass Spectrometry Studies Indicate a Novel Topology for the Glycine Receptor. J. Biol. Chem. 2000, 275(18), 1368313689 .
16. Calabresi, L.; Tedeschi, G.; Treu, C.; Ronchi, S.; Galbiati, D.; Airoldi, S.; Sirtori, C. R.; Marcel, Y.; Franceschini, G. Limited Proteolysis of a Disulfide-Linked ApoA-I Dimer in Reconstituted HDL. J. Lipid Res. 2001, 42(6), 935-942.

17. Bothner, B.; Dong, X. F.; Bibbs, L.; Johnson, J. E.; Siuzdak, G. Evidence of Viral Capsid Dynamics Using Limited Proteolysis and Mass Spectrometry. J. Biol. Chem. 1998, 273(2), 673-676.

18. Aghajanian, S.; Hovsepyan, M.; Geoghegan, K. F.; Chrunyk, B. A.; Engel, P. C. A Thermally Sensitive Loop in Clostridial Glutamate Dehydrogenase Detected by Limited Proteolysis. J. Biol. Chem. 2003, 278(2), 1067-1074.

19. Shields, S. J.; Oyeyemi, O.; Lightstone, F. C.; Balhorn, R. Mass Spectrometry and Non-Covalent Protein-Ligand Complexes: Confirmation of Binding Sites and Changes in Tertiary Structure. J. Am. Soc. Mass Spectrom. 2003, 14(5), 460-470.

20. Schlosser, A.; Klockow, B.; Manstein, D. J.; Lehmann, W. D. Analysis of Post-Translational Modification and Characterization of the Domain Structure of Dynamin a from Dictyostelium Discoideum. J. Mass Spectrom. 2003, 38(3), 277-282.

21. D'Ambrosio, C.; Talamo, F.; Vitale, R. M.; Amodeo, P.; Tell, G.; Ferrara, L.; Scaloni, A. Probing the Dimeric Structure of Porcine Aminoacylase 1 by Mass Spectrometric and Modeling Procedures. Biochemistry 2003, 42(15), 4430-4443.

22. Seielstad, D. A.; Carlson, K. E.; Kushner, P. J.; Greene, G. L.; Katzenellenbogen, J. A. Analysis of the Structural Core of the Human Estrogen Receptor Ligand Binding Domain by Selective Proteolysis/Mass Spectrometric Analysis. Biochemistry 1995, 34(39), 12605-12615.

23. Rudiger, A.; Rudiger, M.; Weber, K.; Schomburg, D. Characterization of Post-Translational Modifications of Brain Tubulin by Matrix-Assisted Laser Desorption/Ionization Mass Spectrometry: Direct One-Step Analysis of a Limited Subtilisin Digest. Anal. Biochem. 1995, 224(2), 532-537.

24. Hamuro, Y.; Burns, L.; Canaves, J.; Hoffman, R.; Taylor, S.; Woods, V. Domain Organization of D-AKAP-2 Revealed by Enhanced Deuterium Exchange-Mass Spectrometry (DXMS). J. Mol. Biol. 2002, 321(4), 703-714.

25. Gervasoni, P.; Staudenmann, W.; James, P.; Pluckthun, A. Identification of the Binding Surface on $\beta$-Lactamase for Groel by Limited Proteolysis and MALDI-Mass Spectrometry. Biochemistry 1998, 37(33), 11660-11669.

26. Bronsoms, S.; Villanueva, J.; Canals, F.; Querol, E.; Aviles, F. X. Analysis of the Effect of Potato Carboxypeptidase Inhibitor Pro-Sequence on the Folding of the Mature Protein. Eur. J. Biochem. 2003, 270(17), 3641-3650.

27. Cohen, S. L.; Ferre-D'Amare, A. R.; Burley, S. K.; Chait, B. T. Probing the Solution Structure of the DNA-Binding Protein Max by a Combination of Proteolysis and Mass Spectrometry. Prot. Sci. 1995, 4(6), 1088-1099.

28. Vinci, F.; Couprie, J.; Pucci, P.; Quemeneur, E.; Moutiez, M. Description of the Topographical Changes Associated to the Different Stages of the DSBA Catalytic Cycle. Prot. Sci. 2002, 11(7), 1600-1612.

29. Persson, U.; Souchelnytskyi, S.; Franzen, P.; Miyazono, K.; ten Dijke, P.; Heldin, C. H. Transforming Growth Factor (TGF- $\beta$ )Specific Signaling by Chimeric TGF- $\beta$ Type II Receptor with Intracellular Domain of Activin Type IIb Receptor. J. Biol. Chem. 1997, 272(34), 21187-21194. 